\title{
0 \\ Microdialysis to optimize cord perfusion and drug delivery in spinal cord injury

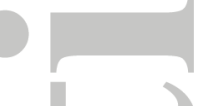 \\ ${ }^{1}$ Isaac Phang MRCS, ${ }^{2}$ Argyro Zoumprouli MD, ${ }^{1}$ Marios C. Papadopoulos MD FRCS(SN), \\ ${ }^{1}$ Samira Saadoun PhD \\ ${ }^{1}$ Academic Neurosurgery Unit, St. George's, University of London, London SW17 0RE, UK \\ ${ }^{2}$ Neurointensive Care Unit, St. George's Hospital, London SW17 0QT, UK
}

Correspondence to: Dr. S. Saadoun, Room 0.136 Jenner Wing, St. George's, University of

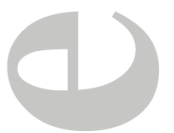

Running head:

Word count:
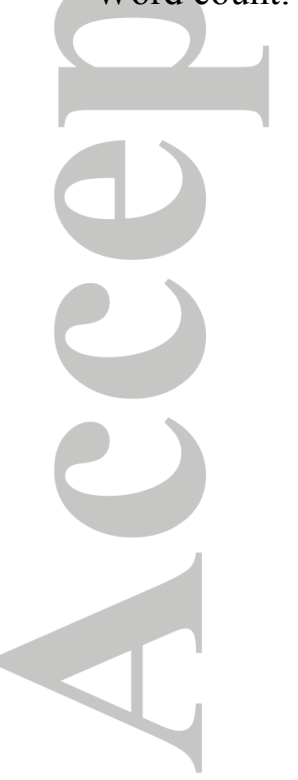

This article has been accepted for publication and undergone full peer review but has not been through the copyediting, typesetting, pagination and proofreading process which may lead to differences between this version and the Version of Record. Please cite this article as an 'Accepted Article', doi: 10.1002/ana.24750 


\section{ABSTRACT}

OBJECTIVE: There is lack of monitoring from the injury site to guide management of patients with acute traumatic spinal cord injury. Here we describe a bedside microdialysis monitoring technique for optimizing spinal cord perfusion and drug delivery at the injury site.

METHODS: 14 patients were recruited within 72 hours of severe spinal cord injury. We inserted intradurally at the injury site a pressure probe, to monitor continuously spinal cord perfusion pressure, and a microdialysis catheter, to monitor hourly glycerol, glutamate, glucose, lactate and pyruvate. The pressure probe and microdialysis catheter were placed on the surface of the injured cord.

RESULTS: Microdialysis monitoring did not cause serious complications. Spinal cord perfusion pressure $90-100 \mathrm{mmHg}$ and tissue glucose $>4.5 \mathrm{mM}$ minimized metabolic derangement at the injury site. Increasing spinal cord perfusion pressure by $\sim 10 \mathrm{mmHg}$, increased the entry of intravenously administered dexamethasone at the injury site three-fold.

INTERPRETATION: This study determined the optimum spinal cord perfusion pressure and optimum tissue glucose concentration at the injury site. We also identified spinal cord perfusion pressure as a key determinant of drug entry into the injured spinal cord. Our findings challenge current guidelines, which recommend maintaining mean arterial pressure at $85-90 \mathrm{mmHg}$ for a week after spinal cord injury. We propose that future drug trials for spinal cord injury include pressure and microdialysis monitoring to optimize spinal cord perfusion and maximize drug delivery at the injury site. 


\section{INTRODUCTION}

Traumatic spinal cord injury (TSCI) is a devastating condition that, each year, affects about $15-40$ people per million ${ }^{1}$. The surgical, anesthetic and neurointensive care unit management of patients with acute TSCI is variable ${ }^{2,3}$. To guide management, we recently developed a novel technique to continuously monitor intraspinal pressure (ISP) at the injury site and compute spinal cord perfusion pressure $(\mathrm{SCPP})^{4,5}$. Intraoperatively, a pressure probe is inserted subdurally to measure ISP at the injury site, which is the same as intraparenchymal ISP $^{6}$. Monitoring ISP and SCPP for up to a week is safe based on a review of 42 patients ${ }^{7}$. Data obtained from human ${ }^{8,9}$ and mouse ${ }^{10}$ studies show that, after TSCI, the injured spinal cord swells and becomes compressed against the dura. Three intradural compartments form (above, at and below the injury site) each with a different ISP ${ }^{11}$. The low SCPP at the injury site ${ }^{5,8}$ suggests that the injured spinal cord is ischemic. We showed that SCPP can be increased by increasing the mean arterial pressure with inotropes ${ }^{5}$ or by reducing ISP with expansion duroplasty ${ }^{8}$. It remains unclear whether increasing SCPP is beneficial for the injured spinal cord.

Microdialysis is widely used to monitor brain metabolism in brain-injured patients ${ }^{12}$. Microdialysis catheters monitor hourly the levels of brain tissue glucose, lactate, pyruvate, glutamate and glycerol in the extracellular space. Low glucose, high lactate, high lactate-topyruvate ratio (LPR) and high lactate-to-glucose ratio (LGR) indicate metabolic derangement ${ }^{13-15}$. Glutamate is released during ischemia and causes neuronal death (excitotoxicity) whereas glycerol is a marker for cell membrane lipid degradation ${ }^{12}$. Microdialysis has also been used to quantify penetration of drugs including vigabatrin ${ }^{16}$, metronidazole ${ }^{17}$ and cefotaxime ${ }^{18}$ into injured human brain. Microdialysis monitoring has not previously been used in TSCI patients. Here, we describe a simple technique for simultaneous bedside monitoring of SCPP and microdialysis at the injury site. This technique 
was used to determine the SCPP and tissue glucose level that minimize the metabolic derangement at the injury site; these are termed the optimal SCPP and the optimal tissue glucose level, respectively. We also determined the effect of altering SCPP on the entry of intravenously administered drugs into the damaged spinal cord.

\section{MATERIALS AND METHODS}

Institutional research board approval. Approval for the Injured Spinal Cord Pressure Evaluation (ISCoPE) study, including the patient information sheet and consent form was obtained from the St George's Joint Research Office and the National Research Ethics Service London - St Giles Committee (No. 10/H0807/23). The study conforms with The Code of Ethics of the World Medical Association (Declaration of Helsinki).

Patient recruitment. Inclusion criteria are: 1. Severe TSCI defined as American spinal injuries association Impairment Scale (AIS) grade A, B or C; 2. Age 18 - 70 years; 3. Timing between TSCI and surgery not more than 72 hours. Exclusion criteria are: 1. Patient unable to consent; 2. Other major injuries or co-morbidities; 3 Penetrating TSCI. Surgery and early management took place at the neurosurgery unit of St. George's Hospital. Recruitment into the study was discussed with all eligible patients and their families on admission, the patient information sheet was given and the consent form was signed by each patient. Recruitment for microdialysis monitoring was between May 2014 and September 2015.

Insertion of ISP probe and microdialysis catheters. Following bony realignment and posterior fixation, the ISP probe (Codman Microsensor Transducer ${ }^{\circledR}$, Depuy Synthes, Leeds, UK) and microdialysis catheter(s) (CMA61, CMA Microdialysis AB, Sweden) were tunnelled through skin into the wound cavity. We chose the CMA61 microdialysis catheter, 
which has a $3 \mathrm{~cm}$ long membrane, long shaft and $20 \mathrm{kDa}$ cut-off rather than the CMA70 or CMA71 brain catheters, which are too short to tunnel for spinal cord monitoring. Details of the CMA61 catheter are in the Supplement. Under the operating microscope, the dura and arachnoid membranes were opened one level below the injury (Fig. 1A). The ISP probe was inserted through the durotomy and placed under the arachnoid on the spinal cord surface. We inserted a microdialysis catheter at the injury site in 14 patients. The microdialysis catheter was inserted until the tip was at the site of maximal spinal cord swelling. The dural opening was sutured and supplemented with fibrin glue (Tisseel ${ }^{\circledR}$, Baxter, UK). ISP probes and microdialysis catheters were secured to the skin with silk sutures. A wound drain was allowed to drain under gravity for a week. Patients were given 5,000 units dalteparin daily starting on the day after the surgery. Dalteparin was omitted 24 hours before removing the probes and wound drain.

ISP and SCPP monitoring. The ISP probe was connected to a Codman ICP box linked via a ML221 amplifier to a PowerLab running LabChart v.7.3.5 (AD Instruments, Oxford, UK). Arterial blood pressure was recorded from a radial artery catheter connected to the Philips Intellivue MX800 bedside monitoring system (Philips, Guildford, UK) in turn connected to the PowerLab system. The ISP and arterial blood pressure signals were sampled at $1 \mathrm{kHz}$ for up to seven days. LabChart was used to analyse the signals and compute SCPP, defined as mean arterial pressure minus ISP.

Microdialysis monitoring. Microdialysis monitoring was started postoperatively in the neurointensive care unit. CNS perfusion fluid (CMA Microdialysis AB, Sweden) was perfused at a rate of $0.3 \mu \mathrm{L} / \mathrm{min}$, unless otherwise stated, using the CMA106 or CMA107 infusion pump (CMA Microdialysis AB, Sweden). The transit time from the microdialysis 
membrane to the vial collection chamber is 24 minutes. Microdialysis vials were changed hourly, stored at $4{ }^{\circ} \mathrm{C}$, and batched analysed up to 24 hours later using ISCUS Flex (CMA Microdialysis AB, Sweden). The first two samples from each patient were discarded to allow priming of the microdialysis catheter and stabilisation of the metabolite concentrations. All samples were analyzed for glucose, lactate, pyruvate, glycerol and glutamate. LPR and LGR were calculated. 100-fold changes in metabolite concentration, compared with the preceding hour, were excluded from analysis. After analysis, samples were stored at $-80{ }^{\circ} \mathrm{C}$.

Patient assessments. Neurological examinations were done on admission and at two weeks postoperatively, i.e. before discharge to the rehabilitation facility. A CT and MRI of the whole spine were done on admission. Another CT was done within 48 hours of surgery to check screw and probe positions (Fig. 1B). MRI of the injured spinal cord was done at about 2 weeks and at $6-12$ months after surgery.

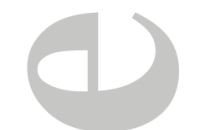

Metabolite recovery. The extrapolation to zero flow method was used to determine metabolite recoveries ${ }^{19}$. Data were collected on the last day of monitoring when metabolite levels were stable. Flow rate was changed every 2 hours and samples were collected every 30 minutes. The first sample of each 2-hour period was discarded to allow for the transit time of the microdialysis fluid from the exchange membrane to the vial. We used flow rates of 0.3 , 1.0, 2.0 and $5.0 \mu \mathrm{L} / \mathrm{min}$. The experiment was repeated in three patients and the following equation was fitted to the data: $R=100 \times[1-\exp (-K / f)]$, where $R$ is metabolite recovery, $K$ is exchange membrane surface area times mass transport coefficient and $f$ is flow rate ${ }^{19}$.

Dexamethasone assay. In three TSCI patients, a bolus of $4 \mathrm{mg}$ dexamethasone was given intravenously. Venous blood was withdrawn before giving dexamethasone ( $0 \mathrm{~min})$ and at 10 
minutes, 1, 2, 4 and 8 hours after dexamethasone administration. Blood was allowed to clot for 30 minutes at room temperature, tubes were centrifuged at 7,000 revolutions per minute for 10 minutes and sera were collected and stored at $-80^{\circ} \mathrm{C}$. In each patient, a microdialysis catheter at the injury site was perfused at $0.3 \mu \mathrm{L} / \mathrm{min}$ and samples were collected hourly. The experiment was done with SCPP kept high. At 24 hours later, the experiment was repeated with SCPP kept low. Serum and microdialysis samples were analysed using an enzymelinked immunosorbent assay kit (IDELISA-F016, ID-Labs, London, ON, Canada) according to the manufacturer's instructions. For serum dexamethasone concentration, we fitted the exponential decay equation $D_{t}=D_{o} \times \exp (-\lambda t)$ where $D_{t}$ is dexamethasone concentration at time $t, D_{o}$ is dexamethasone concentration at time 0 and $\lambda$ is the exponential decay constant. Serum dexamethasone half-life was calculated as $t_{1 / 2}=\ln (2) / \lambda$. We determined the Area Under the Curve at $0-8$ hours $\left(\mathrm{AUC}_{0-8 \mathrm{~h}}\right)$ for serum and spinal cord dexamethasone.

Statistics. We used http://mycurvefit.com to fit $R=100 \times[1-\exp (-K / f)]$ (extrapolation to zero flow) and $D_{t}=D_{o} \times \exp (-\lambda t)$ (dexamethasone kinetics) to the data. Two-way ANOVA (XLSTAT Premium, Addinsoft, New York, USA) was used to model each metabolite concentration (first factor injury site $v s$. below and second factor hours, or first factor AIS A $v s . \mathrm{B} / \mathrm{C}$ and second factor hours) and dexamethasone concentration (first factor high $v s$. low SCPP and second factor time). Statistical significance was $P<0.05^{*}, 0.01^{* *}, 0.005^{\dagger}$, $0.001^{\dagger \dagger}, 0.0005^{\#}, 0.0001^{\# \#}$.

\section{RESULTS}

Patient details. We recruited 14 consecutively admitted TSCI patients with mean age 38 years (Table 1). After surgery, patients were kept in the neurointensive care unit throughout the monitoring period. Most (92.9\%) were male, most (57.1\%) had thoracic injuries and

\section{John Wiley \& Sons}

This article is protected by copyright. All rights reserved. 
most $(71.4 \%)$ had no motor or sensory response below the level of injury on admission (AIS

A). TSCI resulted from a fall in $42.9 \%$ and a road traffic accident in $57.1 \%$. Mean time from injury to surgery was 1.5 days after TSCI. $71.4 \%$ patients had posterior spinal fixation with laminectomy and $28.6 \%$ had posterior fixation without laminectomy. The duration of microdialysis monitoring ranged from less than a day to 6 days. On average, patients had microdialysis monitoring for 4 days. Within 2 weeks of surgery, $14.3 \%$ patients improved from AIS A to B; AIS grade did not worsen in any patient.

Metabolite recovery. Plots of metabolite concentration at different flow rates are in Fig. 1C. For each metabolite, there was excellent goodness-of-fit $(r<-0.95)$ with the theoretical curve. Relative recoveries were calculated from the theoretical curves as the metabolite concentration at $0.3 \mu \mathrm{L} /$ min divided by the estimated metabolite concentration at no flow. Our data indicate relative recoveries at $88-100 \%$ (Fig. 1D).

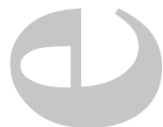

SCPP vs. metabolite levels. During the microdialysis monitoring period the average SCPP of all patients was $60-80 \mathrm{mmHg}$ (Fig. 2A). Figs. 2B-E show the average daily metabolite concentrations, LPR and LGR at the injury site at different SCPPs. SCPP $90-100 \mathrm{mmHg}$ was associated with the least abnormal metabolic profile i.e. lowest glutamate, highest glucose as well as lowest LPR and LGR. Below $90-100 \mathrm{mmHg}$ the metabolism of the injury site progressively worsened, i.e. negative correlation between SCPP vs. glutamate, LPR or LGR and positive correlation between SCPP vs. glucose. SCPP $>100 \mathrm{mmHg}$ was associated with more deranged metabolism at the injury site than SCPP $90-100 \mathrm{mmHg}$, i.e. higher glutamate, lower glucose, higher LPR and higher LGR. Glycerol concentration was high ( $\sim 55$ $\mathrm{mM})$ with SCPP below $60-70 \mathrm{mmHg}$ and low $(\sim 40 \mathrm{mmM})$ with SCPP $>70 \mathrm{mmHg}$. There was no correlation between average daily metabolite concentrations, LPR or LGR at the 
injury site $v s$. ISP (not shown). We also plotted tissue glucose concentration at the injury site vs. LPR (Fig. 2F). Tissue glucose concentration $>4.5 \mathrm{mM}$ was associated with the lowest LPR; below 4.5 mM, glucose concentration inversely correlated with LPR. Glucose concentration at the injury site was $35.5 \pm 1.0 \%$ of serum glucose. Fig. $2 \mathrm{G}$ shows the relationship between serum glucose and microdialysis glucose. The data suggest that, after TSCI, optimum SCPP is $90-100 \mathrm{mmHg}$ and optimum tissue glucose is $>4.5 \mathrm{mM}$.

Metabolites in complete vs. incomplete injuries. In Fig. 3 we compare average daily metabolite concentrations, LPR and LGR for patients with complete (AIS A) vs. incomplete (AIS B/C) neurological deficit. Metabolism at the injury site was more deranged for AIS A than AIS B/C, with markedly higher glycerol, glutamate, lactate, pyruvate, LPR and LGR as well as lower glucose for AIS A. The differences in metabolite levels between AIS A vs. AIS $\mathrm{B} / \mathrm{C}$ were more marked during the first $72 \mathrm{~h}$ after injury. Within $3-4$ days, glycerol and glutamate concentrations were comparable in AIS A vs. AIS B/C patients, though lactate, pyruvate, LPR and LGR remained higher and glucose remained lower throughout the monitoring period in AIS A than AIS B/C. These findings suggest that the severity of TSCI correlates with the degree of metabolic disturbance at the injury site.

Dexamethasone kinetics. The low and high SCPP time profiles following administration of dexamethasone are in Fig. 4A. Over 8 hours, mean low (SCPP (72 $\pm 1 \mathrm{mmHg}$, mean \pm standard error) was significantly lower than mean high SCPP $(84 \pm 1 \mathrm{mmHg})$. The correlation coefficient of the dexamethasone standards curve was 0.99 and the assay coefficient of variation was $14 \%$. After administration of the $4 \mathrm{mg}$ dexamethasone bolus, serum dexamethasone concentration decayed exponentially with no significant difference in dexamethasone concentrations between high $v$. low SCPP. The correlation coefficient for the 
best-fit exponential decay curve was -1.00 for high and low SCPP (Fig. 4B). The $\mathrm{AUC}_{0-}$ $8 \mathrm{~h}(\mathrm{serum})$ and $\mathrm{t}_{1 / 2}$ for serum dexamethasone were comparable at high $v s$. low SCPP: $\mathrm{AUC}_{0-}$ 8h(serum) was 1,091 vs. $942 \mathrm{ng} . \mathrm{h} / \mathrm{mL}$ and $\mathrm{t}_{1 / 2}$ was 2.1 vs. 2.3 hours. Significantly more dexamethasone entered the injured spinal cord at high vs. low SCPP with $\mathrm{AUC}_{0-8 \mathrm{~h}(\mathrm{cord})}$ at 17 vs. $6 \mathrm{ng} . \mathrm{h} / \mathrm{mL}$ and peak spinal cord concentration at 4.8 vs. $2.0 \mathrm{ng} / \mathrm{mL}$ (Fig. 4C). Only a small proportion of the intravenous dexamethasone entered the spinal cord with $\mathrm{AUC}_{0-}$ $8 \mathrm{~h}$ (cord) $/ \mathrm{AUC}_{0-8 \mathrm{~h}(\text { serum })}=1.62 v s .0 .64 \%$ at high $v s$. low SCPP. Therefore, increasing SCPP markedly increases the entry of dexamethasone from the blood into the injured spinal cord.

Complications. Table 2 summarizes patient complications. On average, patients were followed up for $4.1 \pm 1.1$ months (mean \pm standard error). There was no immediate postoperative deterioration in AIS grade. Probe-related complications, noted in $28.5 \%$ patients, were asymptomatic pseudomeningocele or CSF leak through the probe skin exit site. All pseudomeningoceles resolved spontaneously and the CSF leak stopped after we sutured the skin. We did not observe meningitis, spinal cord damage or hematoma. Complications unrelated to the probes include pneumonia, vertebral artery dissection from the original trauma, superficial wound infection and a sacral pressure ulcer. One patient died from pulmonary embolism after he was discharged. In another T12 AIS B patient the mean arterial pressure dropped to $60 \mathrm{mmHg}$ (SCPP $20 \mathrm{mmHg}$ ) for 35 minutes. He developed arm weakness and the sensory level ascended. When the SCPP was increased with norepinephrine, power in the arms normalized and the sensory level descended. C.

\section{DISCUSSION}

We showed that, in patients with acute TSCI, simultaneous monitoring of SCPP and microdialysis from the injury site is feasible and safe. The microdialysis catheter was easily 
inserted during spinal fixation surgery and was removed in the neurointensive care unit. Complications related to the microdialysis catheter were minor; they include CSF leak from the catheter exit site that resolved by suturing the skin and asymptomatic pseudomeningocele that resolved spontaneously. Since our patient sample is small, complications may appear in a larger sample. Our microdialysis data suggest that the optimal SCPP is $90-100 \mathrm{mmHg}$ and the optimal tissue glucose is more than $4.5 \mathrm{mM}$ because these values minimize the metabolic derangement at the injury site. We also showed that simultaneous monitoring of SCPP and microdialysis could be used to enhance drug entry at the injury site. Based on our current findings and earlier studies ${ }^{4-9,20,21}$, a large-scale study of outcome from TSCI in patients who have monitoring vs. those who do not is warranted.

There are technical nuances. First, a long $(3 \mathrm{~cm})$ exchange membrane was inserted to achieve near complete metabolite recovery at $0.3 \mu \mathrm{L} / \mathrm{min}$ flow rate, compared with a $1 \mathrm{~cm}$ long microdialysis membrane at $0.3 \mu \mathrm{L} / \mathrm{min}$, which only achieves $70 \%$ metabolite recovery ${ }^{22}$. Second, we placed the microdialysis catheter under the arachnoid because diffusion across the arachnoid is limited by inter-cellular tight junctions ${ }^{23}$ and is greatly reduced for hydrophilic substances ${ }^{24}$. Third, we used surface rather than intraparenchymal microdialysis. Surface microdialysis was originally described in pig $\operatorname{liver}^{25}$ and myocardium ${ }^{26}$ with excellent correlations between surface and intraparenchymal levels of lactate, glucose and glycerol. We advocate using surface rather than intraparenchymal microdialysis monitoring to minimize the risk of probe-related hematoma and mechanical spinal cord damage.

We found more profound metabolic derangement in AIS A than AIS B/C patients. These findings suggest that the severity of TSCI correlates with the metabolic derangement at the injury site. A microdialysis study in a pig TSCI model concluded that the metabolic response at the injury site depends on the biomechanical nature of the injury ${ }^{27}$. Further 
studies are required to determine if the metabolic derangement at the injury site accurately predicts AIS grade conversion after TSCI.

Microdialysis was used to characterize the relationship between SCPP, ISP, glucose and metabolism at the injury site. SCPP pressure below $70 \mathrm{mmHg}$ was associated with cell lysis (high glycerol). As SCPP increased to $90-100 \mathrm{mmHg}$, metabolism improved (lower glutamate and lactate, lower LPR and LGR and higher glucose). Interestingly, when SCPP increased above $100 \mathrm{mmHg}$ the metabolic profile worsened (higher glutamate and lactate, higher LPR and LGR and lower glucose). Therefore, not only hypoperfusion (SCPP below 90 $\mathrm{mmHg}$ ), but also hyperperfusion (SCPP above $100 \mathrm{mmHg}$ ) may be detrimental for the injured spinal cord. This is consistent with our earlier report of a U-shaped relationship between the spinal pressure reactivity index and SCPP, which also indicated optimum SCPP around $90 \mathrm{mmHg}^{5,8,21}$. After TSCI, ISP is $20-30 \mathrm{mmHg}$ and therefore SCPP $90-100$ $\mathrm{mmHg}$ corresponds to mean arterial pressure $110-130 \mathrm{mmHg}$. Our findings thus challenge current guidelines, which recommend maintaining mean arterial pressure at $85-90 \mathrm{mmHg}$ for a week after $\mathrm{TSCI}^{28}$. Elevating mean arterial pressure after TSCI requires inotropic support, which is associated with several complications ${ }^{29}$. To reduce inotrope requirements and, therefore, inotrope-related complications when aiming for SCPP $90-100 \mathrm{mmHg}$, expansion duroplasty could be performed to lower the ISP ${ }^{8}$. No clear correlation was found between ISP and metabolite levels at the injury site; therefore, unlike changes in SCPP, changes in ISP may not be detrimental for the injured spinal cord. The microdialysis data also showed that the optimum glucose level at the injury site is more than $4.5 \mathrm{mM}$ because this minimizes the LPR. Since acute hyperglycemia impairs functional improvement after $\mathrm{TSCI}^{30}$, it may be safer to elevate the tissue glucose concentration at the injury site by increasing the SCPP rather than by increasing the serum glucose concentration. Future studies employing 
rapid sampling microdialysis may determine if the optimum SCPP and optimum tissue glucose concentration vary between TSCI patients.

We showed that SCPP is a major determinant of drug entry into the injured spinal cord. The serum half-life of dexamethasone was $2-3$ hours, in agreement with earlier studies $^{31}$. By increasing SCPP by about $10 \mathrm{mmHg}$, we increased dexamethasone entry into the injured spinal cord three-fold. Completed ${ }^{32,33}$ and on-going ${ }^{34,35}$ trials of neuroprotective drugs in TSCI do not monitor SCPP or drug entry at the injury site. Our data suggest that failure to optimize SCPP and drug delivery at the injury site may lead to the erroneous conclusion that the tested drug does not improve outcome after TSCI, even if the drug is neuroprotective. A way to increase drug entry at the injury site is by localized delivery through the microdialysis catheter ${ }^{36}$. Given the spatial limitations of microdialysis, further studies employing several microdialysis catheters are required to compare drug entry at the injury site $v s$. the penumbra.

We conclude that multi-modality monitoring from the injury site after TSCI is helpful for guiding clinical management to reduce secondary damage. Further studies are needed to determine if interventions to optimize SCPP and tissue metabolite levels improve outcome.

\section{ACKNOWLEDGEMENTS}

We thank the neurosurgery departments at St. George's Hospital, King's College Hospital and Hurstwood Park Neurological Centre for help with patient recruitment. We also thank the anesthetic, neurointensive care unit and operating theatre staff at St. George's Hospital for help with catheter insertion and data collection. Supported by research funds from Wings for Life Spinal Cord Research Foundation (SS, MCP), Fletcher Fund (MCP), Neurosciences Research Foundation (MCP, IP) and London Deanery (IP). 


\section{AUTHOR CONTRIBUTIONS}

Conception and design of the study (MCP, SS), patient surgery (MCP, AZ), acquisition and analysis of data (MCP, SS, IP, AZ), drafting the manuscript or figures (IP, MCP, SS).

\section{POTENTIAL CONFLICTS OF INTEREST}

None.
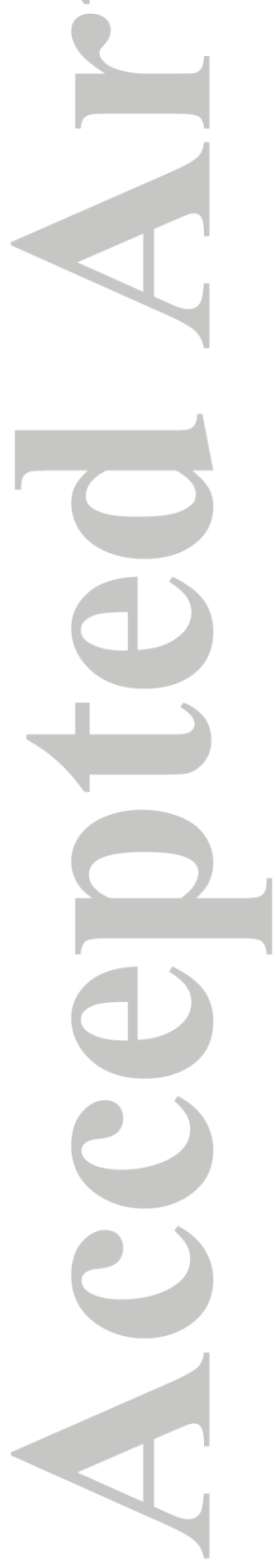

John Willy \& Sons 


\section{REFERENCES}

1. Lee BB, Cripps RA, Fitzharris M, Wing PC. The global map for traumatic spinal cord injury epidemiology: update 2011, global incidence rate. Spinal Cord 2014;52:110-116.

2. Fehlings MG, Rabin D, Sears W, et al. Current practice in the timing of surgical intervention in spinal cord injury. Spine (Phila Pa 1976) 2010;35:S166-173.

3. Werndle MC, Zoumprouli A, Sedgwick P, Papadopoulos MC. Variability in the treatment of acute spinal cord injury in the United Kingdom: results of a national survey. J Neurotrauma 2012;29:880-888.

4. Werndle M, Saadoun S, Phang I, et al. Measurement of intraspinal pressure after spinal cord injury: technical note from the Injured Spinal Cord Pressure Evaluation Study. Acta Neurochir Suppl 2016;122:323-328.

5. Werndle MC, Saadoun S, Phang I, et al. Monitoring of spinal cord perfusion pressure in acute spinal cord injury: initial findings of the injured spinal cord pressure evaluation study. Crit Care Med 2014;42:646-655.

6. Phang I, Papadopoulos MC. Intraspinal Pressure Monitoring in a Patient with Spinal Cord Injury Reveals Different Intradural Compartments: Injured Spinal Cord Pressure Evaluation (ISCoPE) Study. Neurocrit Care 2015;23:763-771.

7. Phang I, Werndle MC, Zoumprouli A, et al. Safety profile and probe placement accuracy of intraspinal pressure monitoring for traumatic spinal cord injury: Injured Spinal Cord Pressure Evaluation study. J Neurosurg Spine 2016:[EPub ahead of print].

8. Phang I, Werndle MC, Saadoun S, et al. Expansion duroplasty improves intraspinal pressure, spinal cord perfusion pressure and vascular pressure reactivity index in patients with traumatic spinal cord injury. J Neurotrauma 2015;32:865-874.

9. Saadoun S, Werndle MC, de Heredia LL, Papadopoulos MC. The dura causes spinal cord compression after spinal cord injury. Br J Neurosurg 2016:[EPub ahead of print]. 
10. Saadoun S, Bell BA, Verkman AS, Papadopoulos MC. Greatly improved neurological outcome after spinal cord compression injury in AQP4-deficient mice. Brain 2008;131:1087-

1098.

11. Papadopoulos MC. Letter: Intrathecal pressure after spinal cord injury. Neurosurgery 2015;77:E500.

12. Hutchinson PJ, Jalloh I, Helmy A, et al. Consensus statement from the 2014 International Microdialysis Forum. Intensive Care Med 2015;41:1517-1528.

13. Carpenter KL, Jalloh I, Hutchinson PJ. Glycolysis and the significance of lactate in traumatic brain injury. Front Neurosci 2015;9:112.

14. Timofeev I, Carpenter KL, Nortje J, et al. Cerebral extracellular chemistry and outcome following traumatic brain injury: a microdialysis study of 223 patients. Brain 2011;134:484-494.

15. Kitagawa R, Yokobori S, Mazzeo AT, Bullock R. Microdialysis in the neurocritical care unit. Neurosurg Clin N Am 2013;24:417-426.

16. Shannon RJ, Timofeev I, Nortje J, et al. Monitoring vigabatrin in head injury patients by cerebral microdialysis: obtaining pharmacokinetic measurements in a neurocritical care setting. Br J Clin Pharmacol 2014;78:981-995.

17. Frasca D, Dahyot-Fizelier C, Adier C, et al. Metronidazole and hydroxymetronidazole central nervous system distribution: 1. microdialysis assessment of brain extracellular fluid concentrations in patients with acute brain injury. Antimicrob Agents Chemother 2014;58:1019-1023.

18. Dahyot-Fizelier C, Frasca D, Gregoire N, et al. Microdialysis study of cefotaxime cerebral distribution in patients with acute brain injury. Antimicrob Agents Chemother 2013;57:2738-2742.

John Wiley \& Sons

This article is protected by copyright. All rights reserved. 
19. Plock N, Kloft C. Microdialysis--theoretical background and recent implementation in applied life-sciences. Eur J Pharm Sci 2005;25:1-24.

20. Phang I, Mada M, Kolias AG, et al. Magnetic Resonance Imaging of the Codman Microsensor Transducer Used for Intraspinal Pressure Monitoring: Findings from the Injured Spinal Cord Pressure Evaluation study. Spine (Phila Pa 1976) 2016;41:E605-E610.

21. Varsos GV, Werndle MC, Czosnyka ZH, et al. Intraspinal pressure and spinal cord perfusion pressure after spinal cord injury: an observational study. J Neurosurg Spine $2015 ; 23: 763-771$.

22. Hutchinson PJ, O'Connell MT, Al-Rawi PG, et al. Clinical cerebral microdialysis: a methodological study. J Neurosurg 2000;93:37-43.

23. Vandenabeele F, Creemers J, Lambrichts I. Ultrastructure of the human spinal arachnoid mater and dura mater. J Anat 1996;189:417-430.

24. Bernards CM, Hill HF. Morphine and alfentanil permeability through the spinal dura, arachnoid and pia mater of dogs and monkeys. Anesthesiology 1990;73:1214-1219.

25. Abrahamsson P, Johansson G, Aberg AM, et al. Outcome of microdialysis sampling on liver surface and parenchyma. J Surg Res 2015;200:480-487.

26. Abrahamsson P, Aberg AM, Johansson G, et al. Detection of myocardial ischaemia using surface microdialysis on the beating heart. Clin Physiol Funct Imaging 2011;31:175181.

27. Okon EB, Streijger F, Lee JHT, et al. Intraparenchymal microdialysis after acute spinal cord injury reveals differential metabolic responses to contusive versus compressive mechanisms of injury. J Neurotrauma 2013;30:1564-1576.

28. Walters BC, Hadley MN, Hurlbert RJ, et al. Guidelines for the management of acute cervical spine and spinal cord injuries: 2013 update. Neurosurgery 2013;60 Suppl 1:82-91. 
29. Inoue T, Manley GT, Patel N, Whetstone WD. Medical and surgical management after spinal cord injury: vasopressor usage, early surgerys, and complications. J Neurotrauma 2014;31:284-291.

30. Kobayakawa K, Kumamaru H, Saiwai H, et al. Acute hyperglycemia impairs functional improvement after spinal cord injury in mice and humans. Sci Transl Med 2014;6:256ra137.

31. Balis FM, Lester CM, Chrousos GP, et al. Differences in cerebrospinal fluid penetration of corticosteroids: possible relationship to the prevention of meningeal leukemia. J Clin Oncol 1987;5:202-207.

32. Bracken MB, Shepard, Holford TR, et al. Administration of methylprednisolone for 24 or 48 hours or tirilazad mesylate for 48 hours in the treatment of acute spinal cord injury. JAMA 1997;277:1597-1604.

33. Bracken MB, Shepard MJ, Hellenbrand KG, et al. Methylprednisolone and neurological function 1 year after spinal cord injury J Neurosurg 1985;63:704-713.

34. Casha S, Zygun D, McGowan MD, et al. Results of a phase II placebo-controlled randomized trial of minocycline in acute spinal cord injury. Brain 2012;135:1224-1236. 35. Fehlings MG, Nakashima H, Nagoshi N, et al. Rationale, design and critical end points for the Riluzole in Acute Spinal Cord Injury Study (RISCIS): a randomized, doubleblinded, placebo-controlled parallel multi-center trial. Spinal Cord 2015;54:8-15.

36. Quan N, Blatteis CM. Microdialysis: a system for localized drug delivery into the brain. Brain Res Bull 1989;22:621-625. 


\section{FIGURE LEGENDS}

Fig. 1. Insertion of microdialysis catheter at injury site and metabolite recovery. A. i. Insertion of ISP probe and microdialysis (MD) catheter under dura and arachnoid. ii. Magnified view of dural entry site. Arrowheads show arachnoid. iii. Sutured dural entry site. iv. Prone patient with cervical TSCI at end of surgery. S, screw; ISP, intraspinal pressure probe. B. Coronal postoperative CT scan through injury site. C. Plots of metabolite concentration $v s$. flow rate and best-fit theoretical curve. Mean \pm standard error. $\mathrm{r}=$ correlation coefficient. D. Percent recovery at flow rate $0.3 \mu \mathrm{L} / \mathrm{min}$.

Fig. 2. Metabolite concentrations at injury site at different SCPPs. A. Mean hourly SCPP vs. time. B. Glycerol, C. Glutamate, D. Glucose, Lactate, Pyruvate, E. LGR and LGR vs. mean hourly SCPP. F. Glucose vs. LPR. G. Serum glucose vs. microdialysis glucose. B - G include the metabolite measurements obtained throughout the monitoring period from all patients. Mean \pm standard error.

\section{Fig. 3. Metabolite concentrations at injury site of AIS A vs. AIS B/C patients. A.} Glycerol, B. Glutamate, C. Glucose, D. Pyruvate, E. Lactate, F. LPR, and G. LGR. Mean \pm standard error. 10 AIS A vs. 4 AIS B/C patients. Mean \pm standard error; $P<0.01^{* *}, 0.0001^{\# \#}$

Fig. 4. Effect of SCPP on penetration of dexamethasone into injured spinal cord. A $4 \mathrm{mg}$ bolus of dexamethasone was given intravenously at time 0 at low SCPP. After $24 \mathrm{~h}$, another 4 $\mathrm{mg}$ dexamethasone bolus was given intravenously at time 0 at high SCPP. A. High and low

SCPP during the experiment. Concentration of dexamethasone B. in serum, and C. at injury site at high and low SCPP. Mean \pm standard error; $N S$, not significant; $P<0.05^{*}, 0.0001^{\# \#}$ 

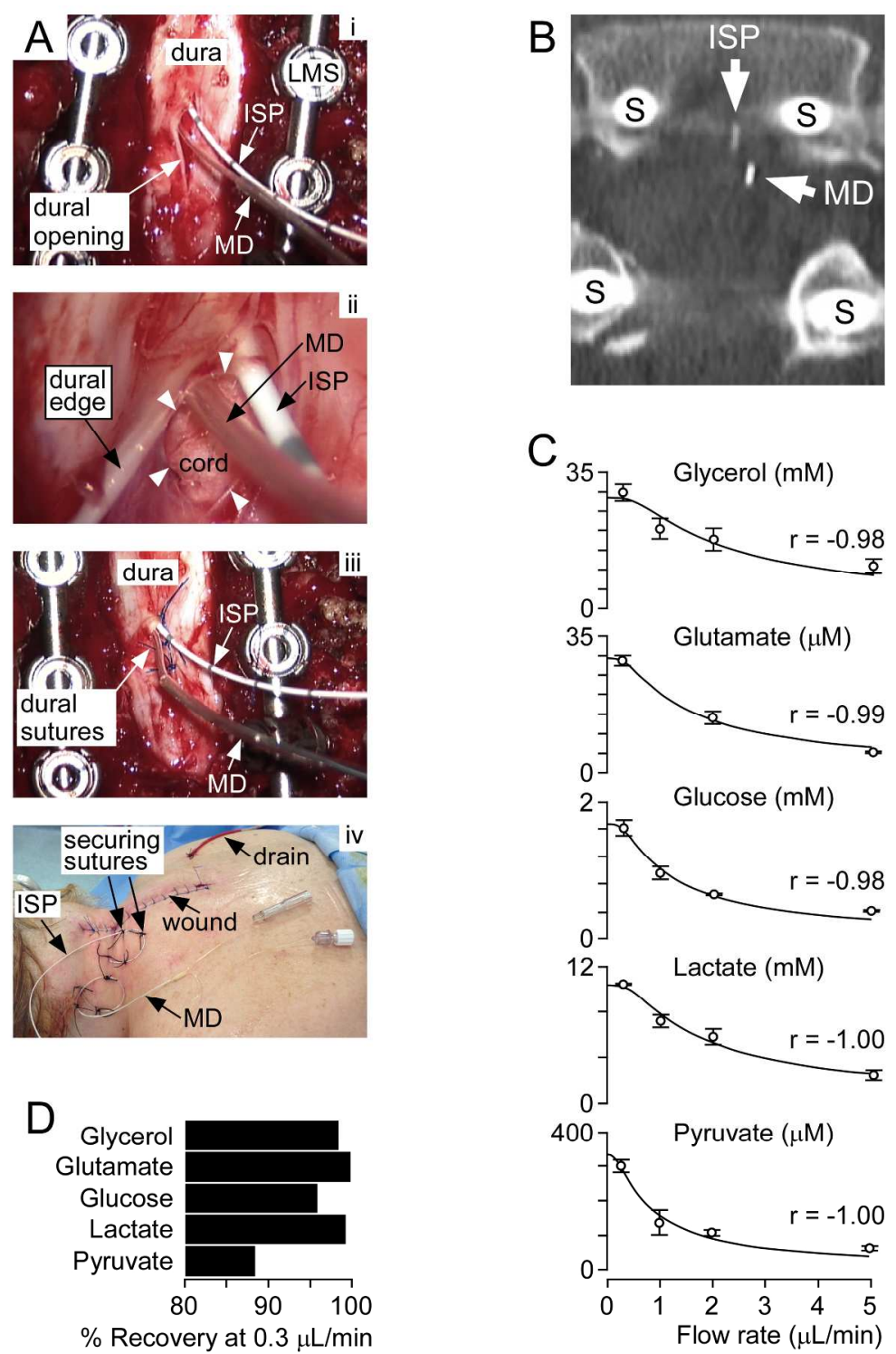

FIGURE 1

$193 \times 273 \mathrm{~mm}(300 \times 300$ DPI $)$ 

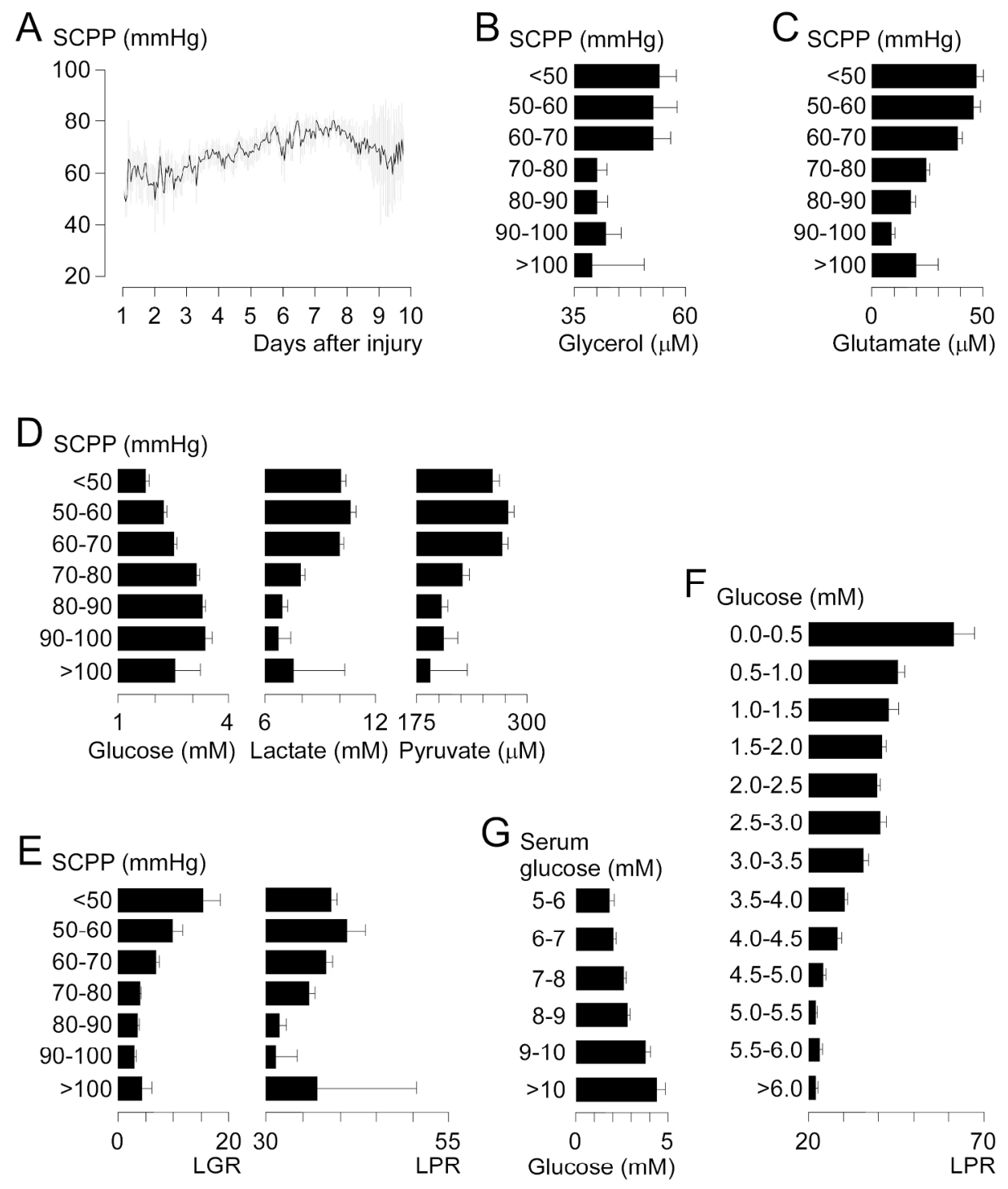

FIGURE 2

$209 \times 267 \mathrm{~mm}(300 \times 300$ DPI $)$

\section{John Wiley \& Sons}

This article is protected by copyright. All rights reserved. 

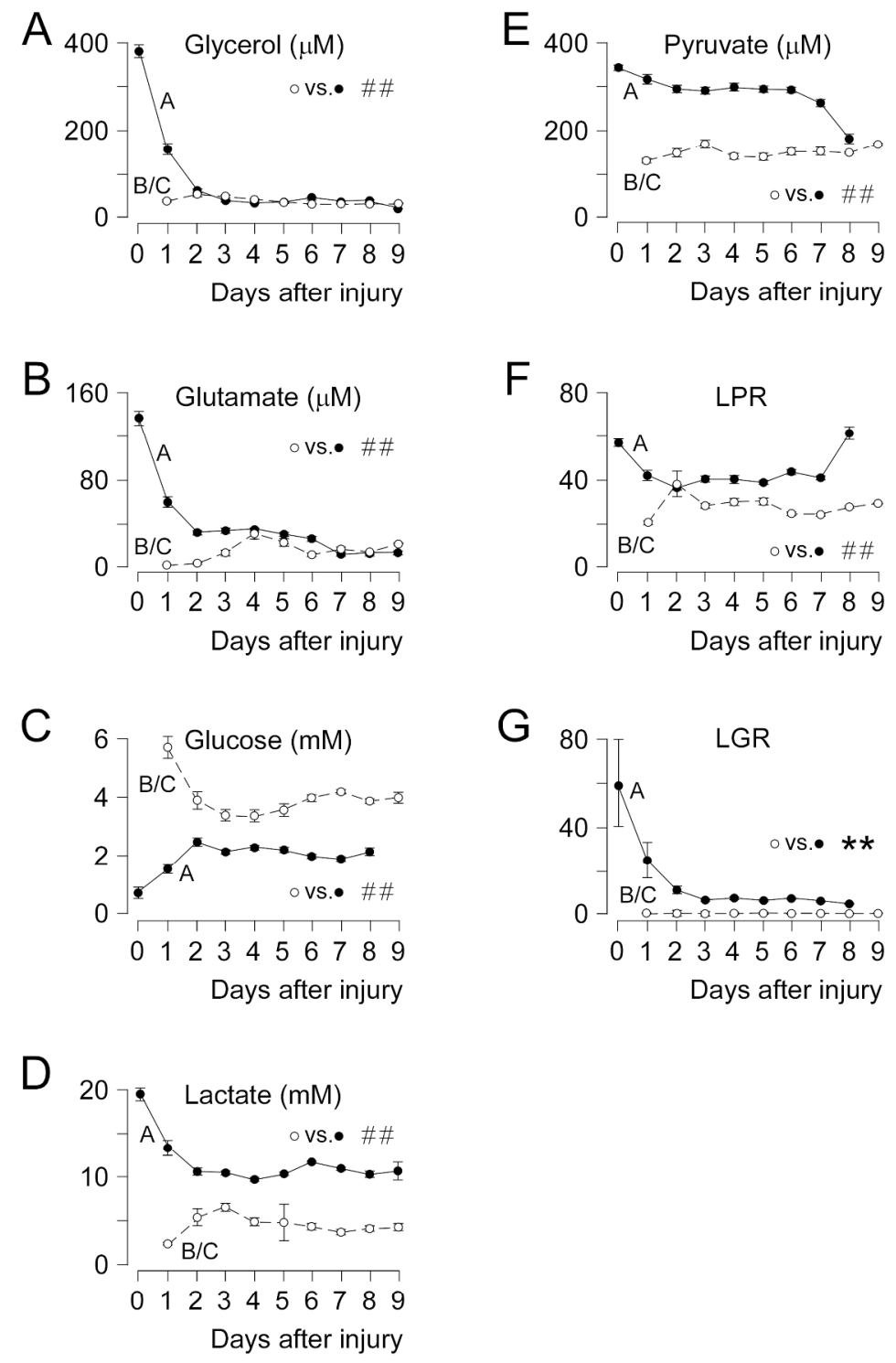

FIGURE 3

$182 \times 264 \mathrm{~mm}(300 \times 300 \mathrm{DPI})$

\section{John Wiley \& Sons}

This article is protected by copyright. All rights reserved. 

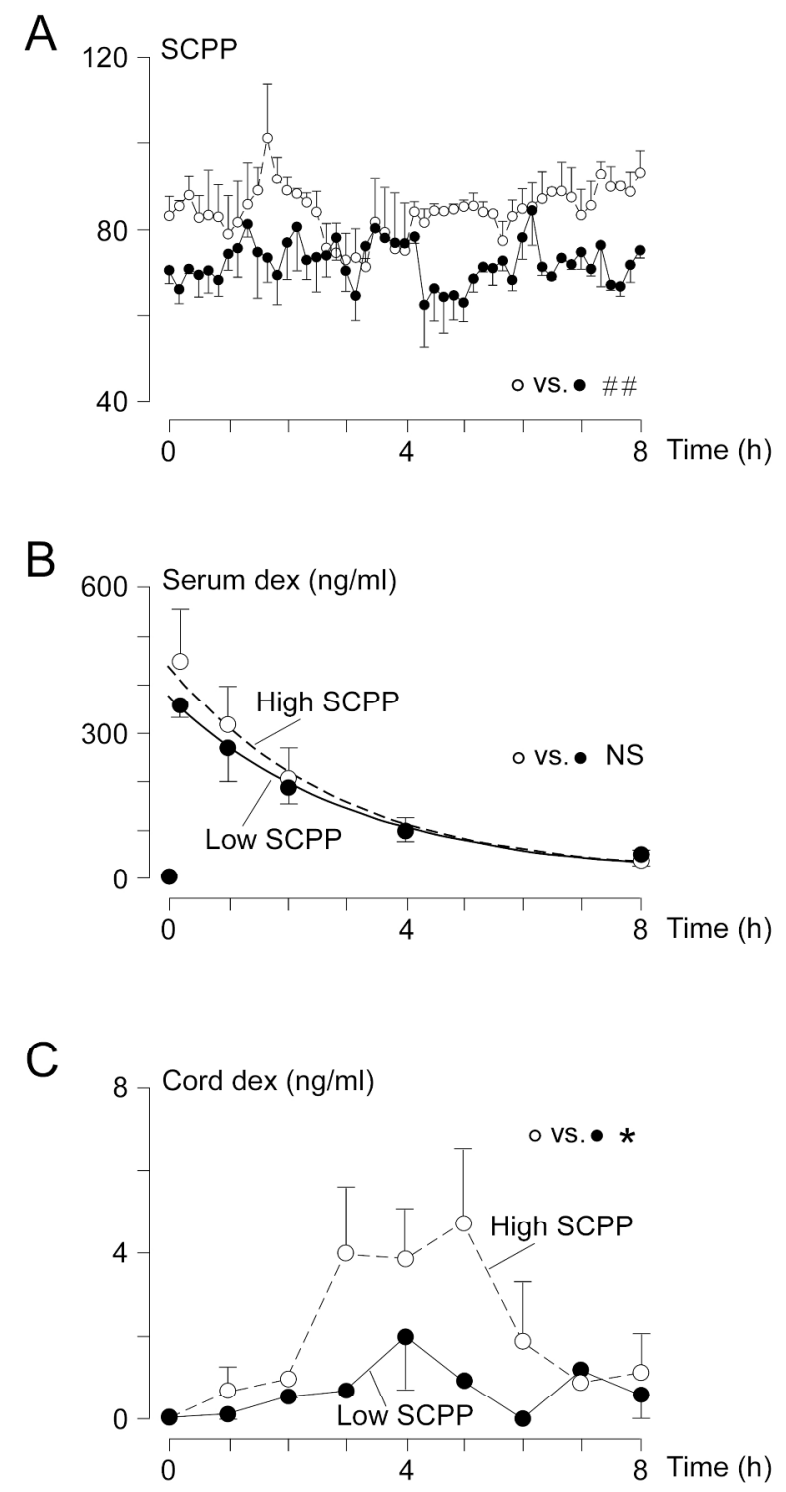

FIGURE 4

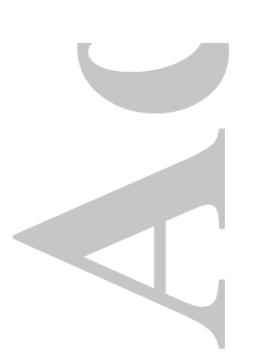

$$
164 \times 270 \mathrm{~mm}(300 \times 300 \mathrm{DPI})
$$

\section{John Wiley \& Sons}

This article is protected by copyright. All rights reserved. 
Table 1. Patient characteristics.

\begin{tabular}{|c|c|}
\hline Number of patients & 14 \\
\hline Mechanism of injury (number of patients) & Fall (6), Road traffic accident (8) \\
\hline in years, mean (range) & $38(19-70)$ \\
\hline Male : female & $13: 1$ \\
\hline Pre-operative AIS (number of patients) & $\mathrm{A}(10), \mathrm{B}(3), \mathrm{C}(1)$ \\
\hline it 2 weeks after surgery (number of patients) & $\mathrm{A}(8), \mathrm{B}(5), \mathrm{C}(1)$ \\
\hline Level of injury (number of patients) & Cervical (6), Thoracic (8) \\
\hline Hours to surgery, mean (range) & $36.5(9-72)$ \\
\hline Laminectomy? (number of patients) & Yes (10), No (4) \\
\hline Hours of microdialysis monitoring, mean (range) & $100(19-145)$ \\
\hline
\end{tabular}
AIS = American spinal injuries association Impairment Scale
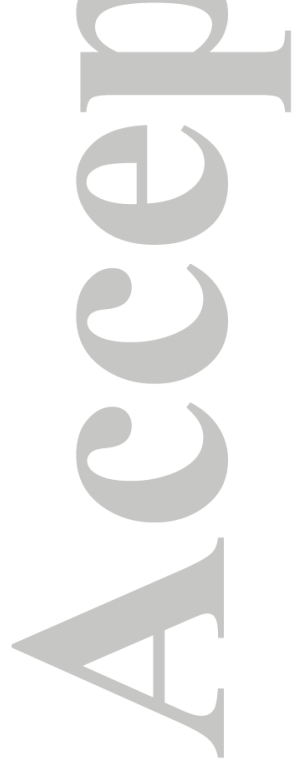
Table 2. Complications in patients who had microdialysis monitoring.

COMPLICATION

NO. OF PATIENTS \%

\section{Associated with microdialysis catheter}

CSF leak ${ }^{\mathrm{a}}$

Asymptomatic pseudomeningocele ${ }^{b}$

Probe associated hematoma ${ }^{\mathrm{c}}$

Meningitis<smiles>C1C2CC12</smiles>

Not associated with microdialysis catheter
1

3

0

0

1

10

1

1

1

1

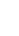

7.1

21.4

0.0

0.0
Hypotension causing spinal cord ischemia ${ }^{\mathrm{f}}$

Superficial wound infection ${ }^{\mathrm{g}}$

Pressure ulcer
7.1

71.4

7.1

7.1

7.1

7.1

${ }^{a}$ Resolved by suturing microdialysis catheter exit site. ${ }^{b}$ MRI at 2 weeks. Pseudomeningoceles resolved on MRI at 6-12 months. ${ }^{c}$ MRI from 11/14 patients. ${ }^{d}$ Patient died from pulmonary embolus at 28 days at a different hospital. ${ }^{e}$ Vertebral artery dissection. ${ }^{f}$ T12 TSCI AIS B. The sensory level ascended from L1 to T5 on day 3 during hypotensive episode (mean arterial pressure $60 \mathrm{mmHg}$, SCPP $20 \mathrm{mmHg}$ for $35 \mathrm{~min}$ ). MRI showed spinal cord ischemia at T4. At 2 weeks the sensory level improved and AIS grade was B. ${ }^{g}$ Resolved with antibiotics.

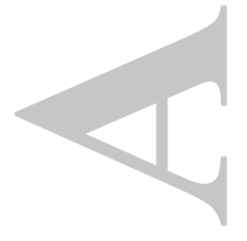

John Wiley \& Sons

This article is protected by copyright. All rights reserved. 